\title{
On the accuracy of creep-damage predictions in thinwalled structures using the finite element method
}

\author{
H. Altenbach, G. Kolarow, 0. K. Morachkovsky, K. Naumenko
}

\begin{abstract}
The constitutive model with a single damage parameter describing creep-damage behaviour of metals with respect to the different sensitivity of the damage process due to tension and compression is incorporated into the ANSYS finite element code by modifying the user defined creep material subroutine. The procedure is verified by comparison with solutions for beams and rectangular plates in bending based on the Ritz method. Various numerical tests show the sensitivity of long-term predictions to the mesh sizes and element types available for the creep analysis of thinwalled structures.
\end{abstract}

1

\section{Introduction}

Engineering structures operating at elevated temperatures such as fossil power plants, chemical plants, reactors, etc. are designed with respect to increased requirements of safety and assurance of long-term reliability. One of the main factors which must be considered in the long-term structural analysis is time-dependent material behaviour coupled with damage evolution (Roche et al., 1992). A powerful tool for the lifetime prediction is the continuum damage mechanics approach which is based on the formulation of constitutive and evolution equations for inelastic strains and material damage. Incorporating these material models into the finite element code predictions of time-dependent stress, strain and damage fields can be performed by numerical solution of nonlinear initialboundary value problems (e.g. Hayhurst, 1994). The first problem arising by creep damage analysis is the formulation of a phenomenological material model that is able to

Received 19 September 1999

H. Altenbach $(\bowtie)$, K. Naumenko Chair of Engineering Mechanics, Department of Engineering Sciences, Martin-Luther-University Halle-Wittenberg, D-06099 Halle, Germany

G. Kolarow

Department of Strength of Materials, TU Sofia, BG-1756 Sofia, Bulgaria

O. K. Morachkovsky

Department of Theoretical Mechanics,

Kharkov State Polytechnical University, ul. Frunze, 21, UKR-310 002 Kharkov, Ukraine

Dedicated to our colleague Prof. Dr.-Ing. Yavuz Başar on the occasion of the sixty fifth birthday describe the sensitivity of creep strain and damage rate to the stress level, stress state, temperature level, environmental effects, etc. Such a model must be able to extrapolate the experimental creep data usually available from uniaxial short-term creep tests and realised for narrow stress ranges to the in-service loading conditions in the real structure. The second problem can be related to the quality of the finite element predictions particularly by analysis of structures with complex shapes.

The structural analysis of thinwalled components (pressure vessels, pipes, pipe bends, etc.) can be performed using the mechanical models of plane stress (strain) states or equations of shell theory. In the case of plane stress (strain) problems numerous finite element simulations considering creep damage effects has been made because of experimental data available for verification. Examples are discussed by Saanouni et al. (1989), Othman et al. (1994), Becker et al. (1994), Murakami and Liu (1995), and Fleig (1996).

Since these examples confirm the ability of finite element simulations to predict stress redistributions and failure times with accuracy enough for engineering applications and can be used as benchmark tests by development of user defined material subroutines incorporating damage evolution a little effort has been made for the analysis of transversely loaded thinwalled structures. Numerical results for rupture times of rectangular plates in bending are given by Bodnar and Chrzanowski (1994), Bialkiewicz and Mika (1995) based on in-house finite element codes and Altenbach and Naumenko (1997) for rectangular plates and Altenbach et al. (1997b) for shells of revolution using special numerical methods. The experimental study of creep damage of plates and shells is very complicated and available experimental data obtained for short times (e.g. Naumenko, 1996) cannot be used for the verification of long-term predictions. However by some simplifications, e.g. for geometry or loading, it is possible to formulate the creep problems which can be solved by direct variational methods using the shape functions defined for the whole domain. Such solutions can be used for the verification of user defined material subroutines and finite element predictions. If mechanical models of thin shells or plates are used in creep analysis the following questions require a special consideration. The first is how sensitive are the long-term predictions of thinwalled structures with predominating bending stresses to the type of elements available in finite element codes for their analysis and to the mesh sizes. The second question is related to the ability of the shell theory used in finite 
element codes (usually the Reissner-Mindlin type theory) to represent the time dependent stress redistributions caused by damage evolution.

This paper presents the results of finite element solutions using the ANSYS code. Basing on the numerical analysis only we discuss the following questions. First we construct special solutions for beams and plates including damage effects based on the Ritz method. These solutions are used for the verification of the ANSYS user defined creep material subroutine which we modify taking into account the damage evolution. Basing on various numerical tests we show the mesh sensitivity of long-term solutions in creep bending of beams and plates. Finally we compare the results for time-dependent deflections and stresses based on different plane stress, shell and solid elements available in the ANSYS code for the plasticity and creep analysis. For the simplicity of numerical studies we limit our discussion to steady state loads and temperatures. Further we do not consider the problems of damage localization which can cause spurious mesh dependency and require special regularization techniques (e.g. Saanouni et al., 1989; Murakami and Liu, 1995).

\section{2}

\section{Constitutive model}

The constitutive model for creep behaviour can be formulated as a set of first order differential equations for the creep rate tensor as a function of the stress tensor, the temperature and possible internal state variables with appropriate evolution equations

$\dot{\boldsymbol{\varepsilon}}^{\mathrm{cr}}=\frac{\partial F\left(\sigma^{\mathrm{eq}}, T ; H_{1}, \ldots, H_{n}, \omega_{1}, \ldots, \omega_{m}\right)}{\partial \boldsymbol{\sigma}}$,

$\dot{H}_{i}=\dot{H}_{i}\left(\sigma_{H}^{\mathrm{eq}}, T ; H_{1}, \ldots, H_{n}, \omega_{1}, \ldots, \omega_{m}\right), \quad i=1, \ldots, n$,

$\dot{\omega}_{k}=\dot{\omega}_{k}\left(\sigma_{\omega}^{\mathrm{eq}}, T ; H_{1}, \ldots, H_{n}, \omega_{1}, \ldots, \omega_{m}\right), \quad k=1, \ldots, m$,

where $\dot{\boldsymbol{\varepsilon}}^{\mathrm{cr}}$ is the creep strain rate tensor, $\boldsymbol{\sigma}$ is the stress tensor, $F$ denotes the creep potential, $H_{i}, \omega_{k}$ are hardening and damage variables. $\sigma_{H}^{\mathrm{eq}}, \sigma^{\mathrm{eq}}$ and $\sigma_{\omega}^{\mathrm{eq}}$ denote the equivalent stresses which control the primary, secondary and tertiary creep, respectively. The internal state variables and the form of the creep potential can be chosen basing on known mechanisms of creep deformation and damage evolution as well as possibilities of experimental measurement and engineering applications (e.g. Chosh and McLean, 1992). The first step by the conventional creep modelling is the formulation of an empirical function describing the sensitivity of minimum creep rate to the stress level and temperature. Using the von Mises type potential the secondary creep constitutive equation can be written as follows (e.g. Altenbach, 1999)

$\dot{\boldsymbol{\varepsilon}}^{\mathrm{cr}}=\frac{3}{2} \frac{f_{\sigma}\left(\sigma^{\mathrm{eq}}\right)}{\sigma^{\mathrm{eq}}} \mathbf{s} f_{T}(T), \quad \sigma^{\mathrm{eq}} \equiv \sigma^{\mathrm{vM}}=\sqrt{\frac{3}{2} \mathbf{s} \cdot \mathbf{s},}$

$\mathbf{s}=\boldsymbol{\sigma}-\frac{1}{3} \operatorname{tr} \boldsymbol{\sigma}$

The mostly used function describing the stress dependency $f_{\sigma}$ is the power law function $f_{\sigma}\left(\sigma^{\mathrm{vM}}\right)=A\left(\sigma^{\mathrm{vM}}\right)^{n}$ with $A$ and $n$ as material constants determined from uniaxial creep tests at constant temperature conditions. The temperature dependence can be described, for instance, by the Arrhenius function (e.g. Nabarro and de Villiers, 1995).

After verification of secondary creep behaviour the minimum creep rate functions can be modified by suitable hardening and damage variables for the description of primary and tertiary creep. Following the classical concept proposed by Kachanov (1986) and Rabotnov (1969) the creep rate equation (2) can be extended by a scalar damage parameter $\omega$ and the damage rate is postulated to be a function of the stress, the temperature and the current damage states. The constitutive and evolution equations for secondary and tertiary creep behaviour can be written as follows

$$
\begin{aligned}
& \dot{\boldsymbol{\varepsilon}}^{\mathrm{cr}}=\frac{3}{2} \frac{f_{\sigma}\left(\sigma^{\mathrm{vM}} / 1-\omega\right)}{\sigma^{\mathrm{vM}}} \mathbf{s} f_{T}(T), \\
& \dot{\omega}=g_{\sigma}\left(\sigma_{\omega}^{\mathrm{eq}}\right) g_{T}(T) g_{\omega}(\omega) .
\end{aligned}
$$

Using the power law functions for $f_{\sigma}, g_{\sigma}$ and $g_{\omega}$ and assuming the temperature to be constant during the creep process the material model can be formulated as (e.g. Leckie and Hayhurst, 1977; Hyde et al., 1996)

$$
\begin{aligned}
& \dot{\boldsymbol{\varepsilon}}^{\mathrm{cr}}=\frac{3}{2} \frac{A\left(\sigma^{\mathrm{vM}}\right)^{n-1}}{(1-\omega)^{n}} \mathbf{s}, \quad \dot{\omega}=\frac{B\left(\left\langle\sigma_{\omega}^{\mathrm{eq}}\right\rangle\right)^{\chi}}{(1-\omega)^{\phi}}, \\
& \sigma_{\omega}^{\mathrm{eq}}=\alpha \sigma_{I}+(1-\alpha) \sigma^{\mathrm{vM}}, \\
& \left\langle\sigma_{\omega}^{\mathrm{eq}}\right\rangle=\sigma_{\omega}^{\mathrm{eq}} \text { for } \sigma_{\omega}^{\mathrm{eq}}>0, \\
& \left\langle\sigma_{\omega}^{\mathrm{eq}}\right\rangle=0 \text { for } \sigma_{\omega}^{\mathrm{eq}} \leq 0, \quad 0 \leq \omega \leq \omega_{*} .
\end{aligned}
$$

Here $\sigma_{I}$ is the maximum principal stress and $B, \chi, \phi, \omega_{*}, \alpha$, are material constants. This model allows a simple description of creep behaviour and has been verified for uniaxial and multiaxial creep of metals and alloys. The coefficient $\alpha$ controls different damage mechanisms. At the same time the model reflect different sensitivity of the damage rate to tension and compression loads. However, the models including another kind of functions for stress and damage dependency (e.g. exponential and hyperbolic functions) are preferred in last years. The approach proposed by Kowalewski et al. (1994) and Perrin and Hayhurst (1994) is based on the introduction of internal variables and corresponding empirical functions of the stress, the temperature, etc. based on the mechanisms of deformation and damage evolution known from the material science and physics of solids. From the numerical studies of final stage of damage evolution and failure propagation Liu and Murakami (1998) propose to replace the power law damage dependence in the evolution equation (4) by the exponential one. The exponential or the hyperbolic functions provide stable solutions particularly for the finite element studies of crack propagation. On the other hand, they can be motivated by metallographical observations. The studies of creep in shells discussed by Altenbach et al. (1997b) using the material model (4) and the model presented by Kowalewski et al. (1994) confirm the ability of mechanism based empirical functions of stress and damage to provide more adequate life-time predictions. In this paper we focus our attention to the mesh sensitivity of finite element based life-time predictions of thinwalled structures 
incorporating the material model (4) into ANSYS finite element code and limit our discussion to the numerical studies of creep behaviour only.

Assuming the elastic properties to be influenced by creep-damage the elastic constitutive equation coupled with damage can be represented as follows

$\sigma_{i j}=C_{i j k l}(\omega)\left(\varepsilon_{k l}-\varepsilon_{k l}^{\mathrm{cr}}\right)$

Considering isotropic material the tensor of elastic properties can be written as (Lemaitre and Chaboche, 1990)

$C_{i j k l}=\frac{E(\omega)}{2\left(1-v^{2}\right)}\left[(1-v)\left(\delta_{i k} \delta_{j l}+\delta_{i l} \delta_{j k}\right)+2 v \delta_{i j} \delta_{k l}\right]$

The function $E(\omega)$ is specified as (Liu et al., 1994)

$E(\omega)=E(1-\omega) \quad$ (fully coupled approach)

$E(\omega)=\left\{\begin{array}{ll}E & \text { if } \quad \omega<\omega_{*} \\ 0 & \text { if } \quad \omega=\omega_{*}\end{array}\right.$ (partly coupled approach),

$E$ and $v$ denote the Young modulus and the Poisson ratio, respectively. For the numerical convenience we will use here the following equation

$E(\omega)=E(1-\zeta \omega), \quad 0 \leq \zeta \leq \frac{1}{\omega_{*}}$.

The constant $\zeta$ controls the influence of the material deterioration on the elastic properties. For $\zeta=0$ we obtain the damage uncoupled classical Hooke law, for $\zeta=1 / \omega_{*}-$ the fully coupled approach that means the zero stiffness $(E=0)$ for the critical damage state.

\section{3}

\section{Creep-damage of beams}

\section{1}

\section{Closed estimations}

The Bernoulli beam is one of the simplest mechanical models which allow to construct a special reference solution and test the finite element software incorporating a user specified material model. First we recall the classical closed solution for uniaxial steady state creep of a beam (e.g. Boyle and Spence, 1983). Neglecting the elastic strain $\varepsilon^{\mathrm{el}} \ll \varepsilon^{\mathrm{cr}}$ and the damage evolution in the material model (4) the constitutive equation for the beam can be written as

$$
\begin{aligned}
& \dot{\varepsilon}(x, z, t) \approx \dot{\varepsilon}^{\mathrm{cr}}(x, z, t) \\
& =-\dot{w}(x, t)^{\prime \prime} z=A|\sigma(x, z, t)|^{n-1} \sigma(x, z, t),
\end{aligned}
$$

where $w(x)$ is the deflection function, $x$ is the beam axis $(0 \leq x \leq l)$ and $z$ is the normal axis $(-h / 2 \leq z \leq h / 2)$. From Eq. (9) follows

$\sigma(x, z, t)=\left[-\frac{\dot{w}(x, t)^{\prime \prime}}{A}\right]^{\frac{1}{n}}|z|^{\frac{1-n}{n}} z$.

The integration over the beam cross section $A_{c}=h b(z)$, which is assumed to be symmetrical, yields

$M(x)=\int_{A_{c}} \sigma z \mathrm{~d} A_{c}=I_{n}\left[-\frac{\dot{w}(x, t)^{\prime \prime}}{A}\right]^{\frac{1}{n}}$ with

$I_{n}=\int_{-h / 2}^{h / 2}|z|^{\frac{n+1}{n}} b(z) \mathrm{d} z=2 \int_{0}^{h / 2}|z|^{\frac{n+1}{n}} b(z) \mathrm{d} z$,

as the generalised moment of inertia.

For a statically determined beam the Eq. (11) yields a solution for the deflection function in steady state creep. As an example we consider a beam simply supported on both edges and loaded by a uniformly distributed force $q$. In this case $M(x)=q x(l-x) / 2$ and the Eq. (11) can be rewritten as

$\dot{w}(x)^{\prime \prime}=-\frac{A}{I_{n}^{n}} \frac{q^{n}}{2^{n}} x^{n}(l-x)^{n}, \quad 0 \leq x \leq l$.

For integer values of the power $n$ the solution is a polynomial of the order $2 n+2$

$\dot{w}(x)=\frac{A}{I_{n}^{n}} \frac{q^{n}}{2^{n}} x(l-x) \sum_{k=0}^{n} \sum_{i=0}^{n+k} \alpha_{k} l^{2 n-i} x^{i}$

$\alpha_{k}=(-1)^{k}\left(\begin{array}{l}n \\ k\end{array}\right) \frac{1}{(n+k+1)(n+k+2)}$.

Comparing with the approximation from the elastic solution

$w(x)=\frac{q}{24 E I} x(x-l)\left(x^{2}-l x-l^{2}\right)$,

with $I$ as the moment of inertia one can conclude that if the creep problem is numerically solved using variational or energy based methods the shape functions for the deflection or deflection rate should contain the polynomial terms of the order $2 n+2$. Further even for analysis of steady state creep using the power low stress dependence an accurate solution cannot be obtained with approximations justified for the elasticity solution.

In order to estimate the solution including damage we make two simplifications that the damage do not accumulate during the transition from the elastic to steady creep state and the damage rate is the same for positive and negative stresses. From the constitutive equation (4) follows

$\sigma(x, z, t)=[1-\omega(x, z, t)]\left[-\frac{\dot{w}(x, t)^{\prime \prime}}{A}\right]^{\frac{1}{n}}|z|^{\frac{1-n}{n}} z$.

The integration over the cross section yields

$$
\begin{aligned}
M(x) & =\int_{-h / 2}^{h / 2} \sigma b(z) z \mathrm{~d} z \\
& =\left[I_{n}-I_{n \omega}(x, t)\right]\left[-\frac{\dot{w}(x, t)^{\prime \prime}}{A}\right]^{\frac{1}{n}}
\end{aligned}
$$

with

$I_{n \omega}(x, t)=2 \int_{0}^{h / 2} \omega(x, z, t)|z|^{\frac{n+1}{n}} b(z) \mathrm{d} z$.

For the example considered we can write the following differential equation 
$\dot{w}(x, t)^{\prime \prime}=-A \frac{q^{n}}{2^{n}} x^{n}(l-x)^{n} \frac{1}{\left[I_{n}-I_{n \omega}(x, t)\right]^{n}}$.

With respect to Eq. (13) the damage evolution equation (4) can be written as

$\dot{\omega}(x, z, t)=B[1-\omega(x, z, t)]^{\chi-\phi}\left[-\frac{\dot{w}(x, t)^{\prime \prime}}{A}|z|\right]^{\frac{\chi}{n}} z$.

Averaging over the cross section yields

$$
\begin{aligned}
\dot{I}_{n \omega}(x, t)= & 2 \int_{0}^{h / 2} \dot{\omega}(x, z, t)|z|^{\frac{n+1}{n}} b(z) \mathrm{d} z \\
= & 2 B\left[-\frac{\dot{w}(x, t)^{\prime \prime}}{A}\right]^{\frac{\chi}{n}} \\
& \times \int_{0}^{h / 2}[1-\omega(x, z, t)]^{\chi-\phi}|z|^{\frac{n+\chi+1}{n}} b(z) \mathrm{d} z .
\end{aligned}
$$

This equation can be simplified for the special case $\chi=\phi$ as

$$
\dot{I}_{n \omega}(x, t)=B\left[-\frac{\dot{w}(x, t)^{\prime \prime}}{A}\right]^{\frac{\chi}{n}} I_{n \chi}
$$

with

$I_{n \chi}=2 \int_{0}^{h / 2}|z|^{\frac{n+\chi+1}{n}} b(z) \mathrm{d} z$.

Using the Eq. (16) we obtain

$\dot{I}_{n \omega}(x, t)=B I_{n \chi} \frac{q^{\chi}}{2 \chi} x^{\chi}(l-x)^{\chi} \frac{1}{\left[I_{n}-I_{n \omega}(x, t)\right]^{\chi}}$.

After the integration with respect to time we get

$$
\begin{aligned}
I_{n} & -I_{n \omega}(x, t) \\
& =I_{n}\left[1-(\chi+1) B I_{n \chi} \frac{q^{\chi}}{2^{\chi} I_{n}^{\chi+1}} x^{\chi}(l-x)^{\chi} t\right]^{\frac{1}{\chi+1}} .
\end{aligned}
$$

Substitution into Eq. (16) yields

$$
\dot{w}(x, t)^{\prime \prime}=-A \frac{q^{n}}{2^{n} I_{n}^{n}} x^{n}(l-x)^{n} f(x, t)
$$

with

$f(x, t)=\left[1-(\chi+1) B I_{n \chi} \frac{q^{\chi}}{2^{\chi} I_{n}^{\chi+1}} x^{\chi}(l-x)^{\chi} t\right]^{-\frac{n}{\chi+1}}$.

From Eq. (15) follows that the condition $I_{n \omega}\left(x_{f}, t_{f}\right)=I_{n}$ is possible in the case of completely damaged cross section $x_{f}$ only. In this case the left part of Eq. (21) vanishes and the function $f\left(x_{f}, t_{f}\right)$ in Eq. (23) tends to infinity for the cross section $x_{f}$. If the numerical study performed until the damage parameter $\omega(x, z, t)$ attains the critical value in a point $P\left(x_{f}, z_{f}\right)$ for $t=t_{*}, I_{n \omega}(x, t)<I_{n}$ holds true and Eq. (23) can be represented as a convergent polynomial series of $x$ for each $0 \leq t \leq t_{*}$. Consequently there exist a convergent solution $\dot{w}(x, t)$ which can be represented as a polynomial series of $x$ containing at least the order $2 n+2$. In the following we confirm these estimations solving the problem by the Ritz method and construct some convergent test solutions.

3.2

\section{Solutions based on the Ritz method}

Starting from Hooke's law (5) applied to the beam bending problem (Pilkey and Wunderlich, 1994)

$$
\begin{aligned}
\sigma & =E(1-\zeta \omega)\left(\varepsilon-\varepsilon^{\mathrm{cr}}\right) \\
& =-E(1-\zeta \omega)\left(-u_{0}^{\prime}-w^{\prime \prime} z+\varepsilon^{\mathrm{cr}}\right),
\end{aligned}
$$

with $u_{0}$ as the axial displacement of the beam centreline, the principle of virtual displacements yields

$$
\begin{aligned}
\int_{V} \sigma \delta \varepsilon \mathrm{d} V= & E \int_{x}\left(I-\omega^{z z z}\right) w^{\prime \prime} \delta w^{\prime \prime} \mathrm{d} x \\
& +E \int_{x}\left(A_{c}-\omega^{z}\right) u_{0}^{\prime} \delta u_{0}^{\prime} \mathrm{d} x \\
& +E \int_{x} \omega^{z z} \delta\left(u_{0}^{\prime} w^{\prime \prime}\right) \mathrm{d} x+E \int_{x} M^{\mathrm{cr}} \delta w^{\prime \prime} \mathrm{d} x \\
& -E \int_{x} N^{\mathrm{cr}} \delta u_{0}^{\prime} \mathrm{d} x \\
= & \int_{x} q \delta w \mathrm{~d} x
\end{aligned}
$$

with

$$
\begin{aligned}
& M^{\mathrm{cr}}=\int_{A_{c}}(1-\zeta \omega) \varepsilon^{\mathrm{cr}} z \mathrm{~d} A_{c}, N^{\mathrm{cr}}=\int_{A_{c}}(1-\zeta \omega) \varepsilon^{\mathrm{cr}} \mathrm{d} A_{c}, \\
& \omega^{z}=\int_{A_{c}} \omega \mathrm{d} A_{c}, \omega^{z z}=\int_{A_{c}} \omega z \mathrm{~d} A_{c}, \omega^{z z z}=\int_{A_{c}} \omega z^{2} \mathrm{~d} A_{c} .
\end{aligned}
$$

Assuming the creep strain and the damage parameter to be known functions of the coordinates $x$ and $z$ for the fixed time $t$ we can formulate the following functional

$$
\begin{aligned}
\Pi_{t}\left(w, u_{0}\right)= & \frac{1}{2} E \int_{x}\left(I-\omega^{z z z}\right) w^{\prime 2} \mathrm{~d} x \\
& +\frac{1}{2} E \int_{x}\left(A_{c}-\omega^{z}\right) u_{0}^{\prime 2} \mathrm{~d} x+E \int_{x} \omega^{z z} u_{0}^{\prime} w^{\prime \prime} \mathrm{d} x \\
& +E \int_{x} M^{\mathrm{cr}} w^{\prime \prime} \mathrm{d} x-E \int_{x} N^{\mathrm{cr}} u_{0}^{\prime} \mathrm{d} x \\
& -\int_{x} q w \mathrm{~d} x .
\end{aligned}
$$

This leads to the problem to find such functions $w$ and $u_{0}$ that yield an extremal value of the functional. The solutions for fixed time $t$ can be represented as

$$
w(x)=a_{0}^{w} \varphi_{0}^{w}(x)+\sum_{i=1}^{N} a_{i}^{w} \varphi_{i}^{w}(x), \quad u_{0}(x)=\sum_{i=0}^{M} a_{i}^{u} \varphi_{i}^{u}(x) .
$$


For the simply supported beam discussed above the shape functions can be formulated as follows:

$\varphi_{0}^{w}(x)=x(x-l)\left(x^{2}-l x-l^{2}\right)$

is the first approximation following from the elastic solution. For $\varphi_{i}^{w}(x)$ we use functions satisfying the boundary conditions $w=0$ and $M=0$ for $x=0$ and $x=l$ :

- polynomials

$$
\varphi_{i}^{w}(x)=x^{i+2}(l-x)^{i+2}
$$

- eigenfunctions of a transverse vibrating beam

$$
\varphi_{i}^{w}(x)=\sin \frac{(2 i-1) \pi x}{l}
$$

Assuming $u_{0}=0$ for the edge $x=0$ the functions $\varphi_{i}^{u}(x)=x^{i+1}$ can be used. Summarising the unknown constant coefficients into the vector $\mathbf{a}^{\mathrm{T}}=\left[\mathbf{a}^{w^{\mathrm{T}}}, \mathbf{a}^{u^{\mathrm{T}}}\right]$ with $\mathbf{a}^{w^{T}}=\left[a_{0}^{w}, a_{i}^{w}\right], i=1, \ldots, N$ and $\mathbf{a}^{u^{\mathrm{T}}}=\left[a_{i}^{u}\right], i=1, \ldots, M$, the Ritz method yields a set of linear algebraic equations

$$
\frac{\partial \mathbf{\Pi}_{t}}{\partial a_{k}}=0, \quad\left[\begin{array}{ll}
\mathbf{R}^{w w} & \mathbf{R}^{w u} \\
\mathbf{R}^{u w} & \mathbf{R}^{u u}
\end{array}\right]\left[\begin{array}{l}
\mathbf{a}^{w} \\
\mathbf{a}^{u}
\end{array}\right]=\left[\begin{array}{l}
\mathbf{f}^{w} \\
\mathbf{f}^{u}
\end{array}\right]
$$

with

$$
\begin{aligned}
R_{k j}^{w w} & =E \int_{0}^{l}\left(I-\omega^{z z z}\right) \varphi_{k}^{w \prime \prime} \varphi_{j}^{w \prime \prime} \mathrm{d} x, \\
k & =1, \ldots, N, \quad j=1, \ldots, M \\
R_{k j}^{w u} & =E \int_{0}^{l} \omega^{z z} \varphi_{k}^{w \prime \prime} \varphi_{j}^{u \prime} \mathrm{d} x, \quad k=1, \ldots, N, j=1, \ldots, M
\end{aligned}
$$$$
R_{k j}^{u u}=E \int_{0}^{l}\left(A_{c}-\omega^{z}\right) \varphi_{k}^{u \prime} \varphi_{j}^{u \prime} \mathrm{d} x,
$$$$
k=1, \ldots, M, \quad j=1, \ldots, M
$$$$
R_{k j}^{u w}=E \int_{0}^{l} \omega^{z z} \varphi_{k}^{u \prime} \varphi_{j}^{w \prime \prime} \mathrm{d} x, \quad k=1, \ldots, M, j=1, \ldots, N
$$$$
f_{k}^{w}=q \int_{0}^{l} \varphi_{k}^{w} \mathrm{~d} x-E \int_{0}^{l} M^{\mathrm{cr}} \varphi_{k}^{w \prime \prime} \mathrm{d} x, \quad k=1, \ldots, N
$$$$
f_{k}^{u}=E \int_{0}^{l} N^{\mathrm{cr}} \varphi_{k}^{u^{\prime}} \mathrm{d} x, \quad k=1, \ldots, M .
$$

After the solution of the system (28), the stress $\sigma(x, z, t)$ can be calculated from the Eq. (24). For the known values of the stress and the damage parameter the constitutive model (4) yields the rates of creep strain and damage for the time $t$. From these the new values for time $t+\Delta t$ are calculated using the implicite time integration procedure

$$
\begin{gathered}
\varepsilon^{\mathrm{cr}}(x, z, t+\Delta t)=\varepsilon^{\mathrm{cr}}(x, z, t)+\Delta t\left[(1-\theta) \dot{\varepsilon}^{\mathrm{cr}}(x, z, t)\right. \\
\left.+\theta \dot{\varepsilon}^{\mathrm{cr}}(x, z, t+\Delta t)\right], \\
\begin{aligned}
\omega(x, z, t+\Delta t)=\omega(x, z, t)+\Delta t[(1-\theta) \dot{d}(x, z, t) \\
+\theta \dot{\omega}(x, z, t+\Delta t)], \\
\varepsilon^{\mathrm{cr}}(x, z, 0)=0, \quad \omega(x, z, 0)=0, \quad \omega(x, z, t)<\omega_{*} .
\end{aligned}
\end{gathered}
$$

For the calculation of the creep force $N^{\mathrm{cr}}$, the creep moment $M^{\text {cr }}$ as well as damage averages $\omega^{z}, \omega^{z z}$ and $\omega^{z z z}$ in Eq. (24) the Gauss method with 9 integration points in the thickness direction is used. For the calculation of the matrices $\mathbf{R}^{m n}$ and the right parts of the equation set (28) the Simpson quadrature rule with $N_{s}$ integration points in the beam axis $x$ is used. The values of creep strain and damage for a current time step $t$ are stored in all integration points along the beam axis and over the thickness direction for calculations in the next time step. The accuracy of the numerical solution depends on the number of shape functions in Eq. (25), on the number of integration points and on the time step size. The sensitivity of creep solutions to time step sizes has been previously studied by Altenbach and Naumenko (1997) on the plate bending problems. In the following numerical examples we will put our attention to the convergence study of the time dependent solution using different number of shape functions in Eq. (24).

As a first example we consider the above discussed simply supported beam with a rectangular cross section $b(z)=$ const. For the calculation we set $q=60 \mathrm{~N} / \mathrm{mm}$, $l=1000 \mathrm{~mm}, h=80 \mathrm{~mm}, b=30 \mathrm{~mm}$ and use the creepdamage material model (4) modified with time-hardening function which allows the description of the primary creep

$\dot{\boldsymbol{\varepsilon}}^{\mathrm{cr}}=\frac{3}{2} \frac{A\left(\sigma^{\mathrm{vM}}\right)^{n-1}}{(1-\omega)^{n}} \mathbf{s} t^{m}, \quad \dot{\omega}=\frac{B\left(\left\langle\sigma_{\omega}^{\mathrm{eq}}\right\rangle\right)^{\chi}}{(1-\omega)^{\phi}} t^{m}$.

In the following we will use the material constants identified by Kowalewski et al. (1994) for an aluminium alloy: $A=3.511 \times 10^{-31} \mathrm{MPa}^{-n} / \mathrm{h}^{m+1}, B=1.960 \times 10^{-23}$ $\mathrm{MPa}^{-\chi} / \mathrm{h}^{m+1}, \chi=8.220, n=11.034, \phi=12.107$, $m=-0.3099$ and $E=7.1 \times 10^{4} \mathrm{MPa}, v=0.3$. Eq. (29) can be transformed into Equation (4) introducing the time variable $\tau=t^{m+1} /(m+1)$. For the first example we neglect the influence of creep damage on elasticity setting $\zeta=0$ in Eq. (8). Further we assume the damage rate to be the same for tensile and compressive loading setting $\alpha=0$ in Eq. (4). Both the assumptions lead to a significant simplification of the numerical procedures. Using the first one we can set $\mathbf{R}^{u w}=\mathbf{R}^{w u}=0$ in Eq. (28) and keep the matrices $\mathbf{R}^{w w}, \mathbf{R}^{u u}$ constant during the time step calculation. The second results to symmetric stress redistributions across the $z$ direction for the arbitrary time step which leads to $N^{\mathrm{cr}}=0$ and $\mathbf{f}^{u}=\mathbf{0}$. Consequently the number of functions in the approximation for the displacement $u_{0}$ has no influence on the numerical solutions.

Figure 1 shows time dependent solutions for maximum deflection and maximum stress obtained by different number of polynomial terms (26) in Eq. (25). The time step solutions are performed until the critical damage is achieved in one of the integration points. The condition of termination of the time step solution is $\omega\left(x_{f}, z_{f}, t_{*}\right)>0.9$, where the integration point $P\left(x_{f}, z_{f}\right)$ can be specified as a point of failure initiation and the time step $t_{*}$ as the time to failure initiation.

Since all approximations of the deflection function used for the Ritz method yield the exact elasticity solution, the life-time predictions are strongly sensitive to the number of shape functions, Fig. 1. Even for the case of a statically determined beam one can conclude that the approximation adjusted for the elastic solution cannot be used for the creep-damage predictions. The difference between the life-time predictions based on an approximation following from the elastic solution (fourth order 

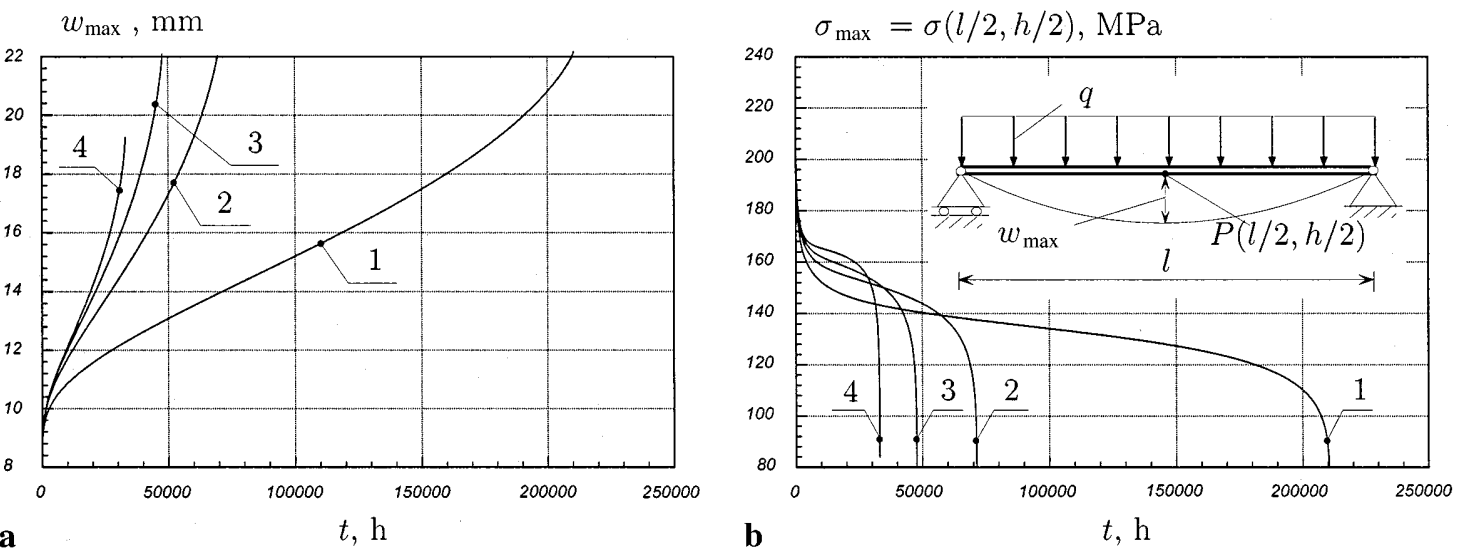

Fig. 1a, b. Solutions for a Bernoulli beam based on the Ritz method using the approximation (25) and polynomial functions (26): a time variation of maximum deflection; $\mathbf{b}$ time variation of maximum stress, 1-approximation using elastic deflection function, $2-N=1,3-N=2,4-N=8$
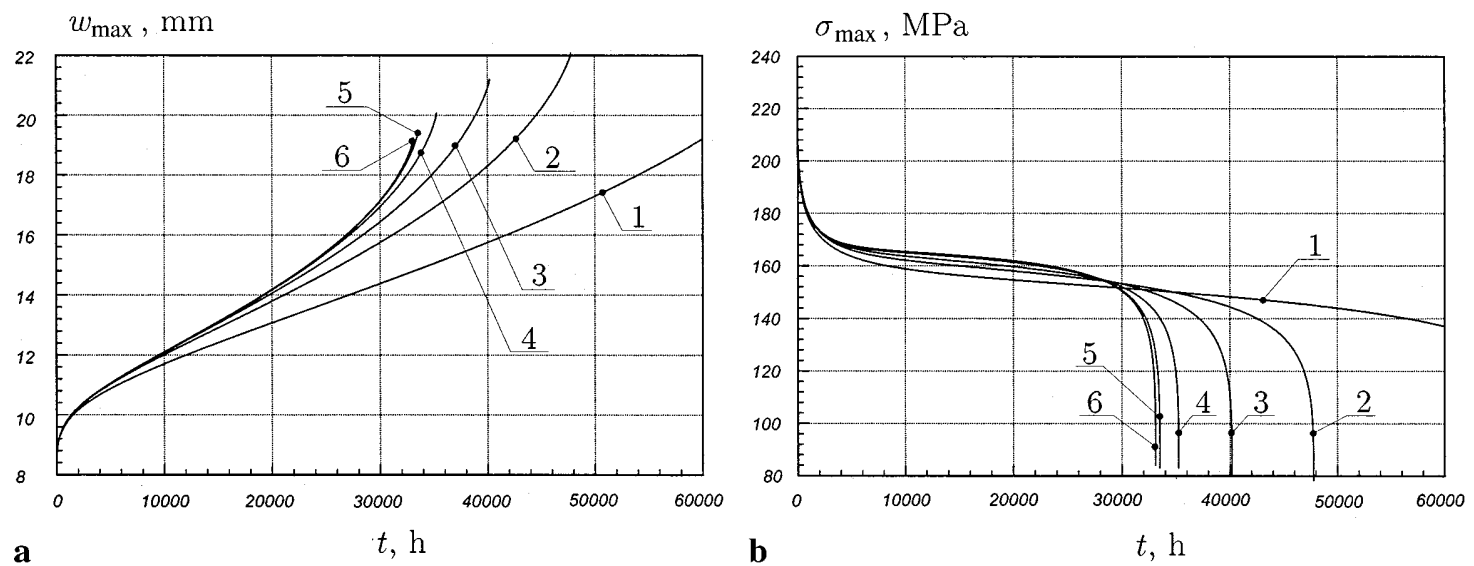

Fig. 2a, b. Convergence of time-dependent solution for a Bernoulli beam using polynomial functions (26): a time variation of maximum deflection; b time variation of maximum stress, $1-N=1,2-N=2,3-N=3,4-N=5,5-N=7,6-N=8$

polynomial), curves 1 , and approximation which follows from above discussed steady state creep solution (polynomial of the order 24, curve 4) is approximately six times. From the Fig. 2 can be seen that the creep-damage solution converges with the increasing number of shape functions. The approximations with $N=5, N=7$ and $N=8$ yield the same solutions for the transition and steady state creep state but differ in the last stage. This is in agreement with the above conclusion that the consideration of damage needs an increased order of approximation in comparison with the steady state creep solution. Since the difference between the cases 4 and 6 (approximately 17\%) is not acceptable comparing with the accuracy of the available material description one can conclude that the order of approximation adjusted for the steady state creep solution can be enough for the numerical life-time predictions using continuum damage mechanics approach. Comparing with the polynomial approximation (26) the eigenfunctions of transverse beam vibration (27) provide a better convergence, Fig. 3. These functions we will use in the analysis of plates in bending below.

In addition to convergence studies, we show the influence of the constant $\zeta$ in Eq. (8) which allows to take into account the drop of elastic stiffness with creep damage accumulation. For the beam considered we compare two convergent solutions obtained by setting $\zeta=0$ and $\zeta=1$ in Eq. (28). The results for the time dependent maximum deflection, maximum stress, as well as creep strain and damage parameter for the integration

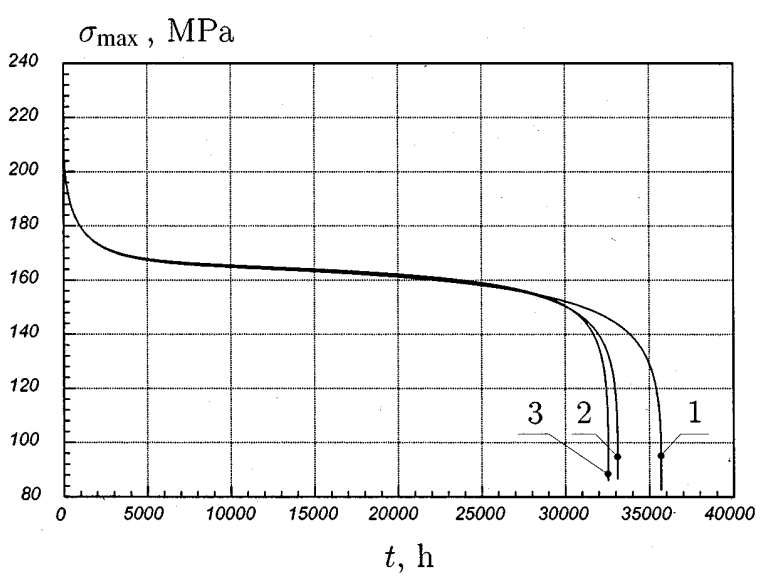

Fig. 3. Convergence of time-dependent solution for a Bernoulli beam using the approximation (25) and eigenfunctions of transverse vibration (27): $1-N=2,2-N=3,3-N=4$ 

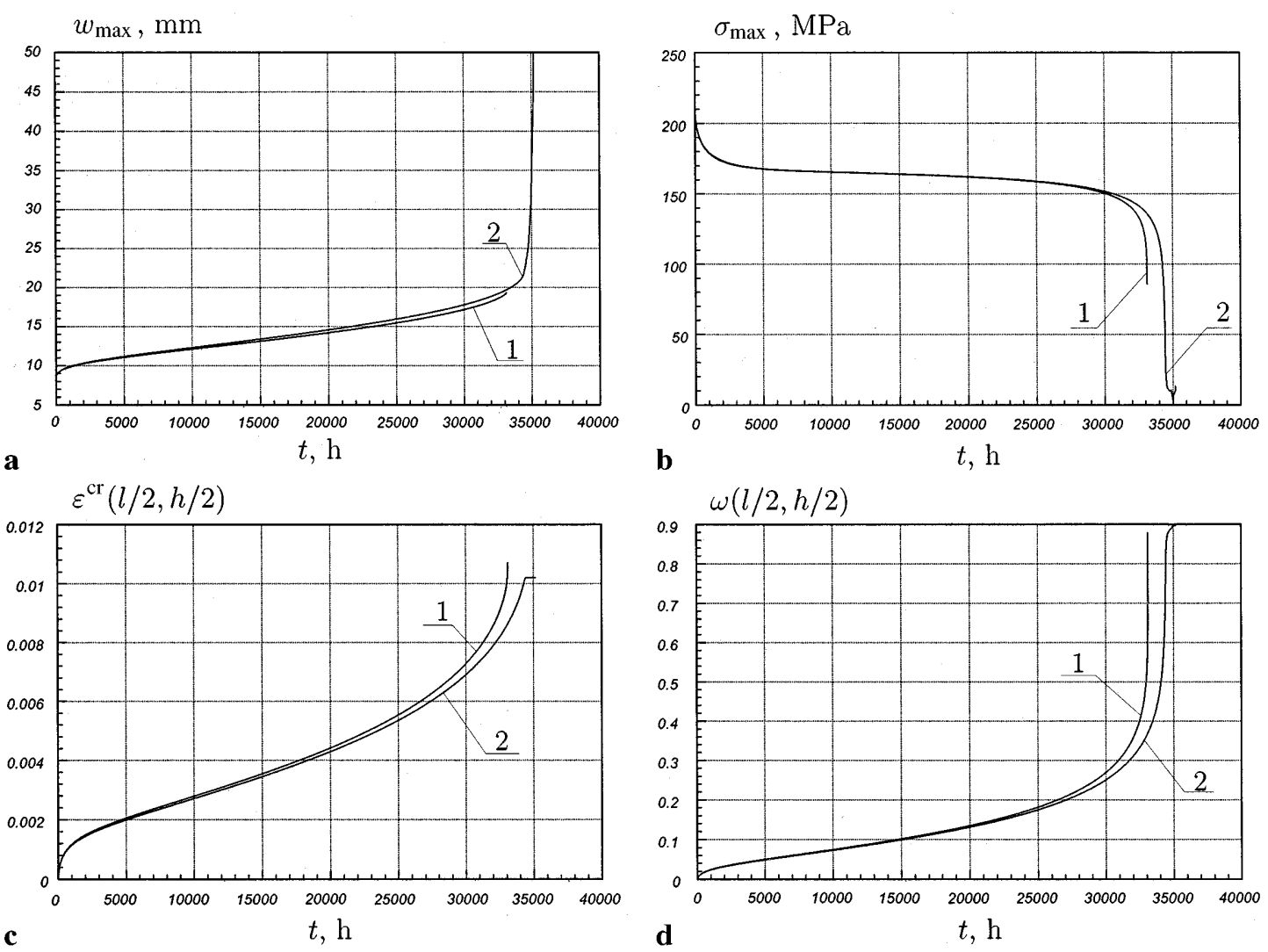

Fig. 4a-d. Influence of the parameter $\zeta$ in Eq. (8) on time variations for: a maximum deflection; $\mathbf{b}$ maximum stress; $\mathbf{c}$ creep strain; d damage parameter, $1-\zeta=0$ uncoupled approach, $2-\zeta=1$ fully coupled approach

point corresponding to $x=l / 2, z=h / 2$ are plotted in Fig. 4. The results for the both cases differ on the last stage before the failure initiation. The fully coupled approach $\zeta=1$ yields the complete stress relaxation and a larger rate of deflection growth for the last stage. The consideration of such effects can be necessary for numerical studies of failure propagation within the framework of continuum damage mechanics (e.g. Saanouni et al., 1989; Murakami and Liu, 1995). But if only the life-time to the failure initiation have to be analysed the uncoupled approach $\zeta=0$ is enough for the numerical predictions. The difference between failure times for both cases is not significant. In addition, it should be noted that by using the coupled approach $\zeta>0$ the computational effort sufficiently increases because the matrices $R$ in Eq. (28) have to be modified on each time or iteration step with the damage field functions obtained in the discretization points. Since the damage localizes at the last stage of calculation it needs a very precise integration (great number of integration points) and a very accurate time step method. This has been previously observed in different finite element calculations (e.g. Saanouni et al., 1989; Murakami and Liu, 1995).

\section{3}

\section{Finite element solution}

Using the ANSYS User Programmable Features we have incorporated the material model (4) into the ANSYS finite element code by modifiying the user creep material subroutine. For details of time integration and equilibrium iteration methods used in ANSYS for creep calculations we refer to ANSYS User's Manual Volume I-IV (1994) and Zienkiewicz and Taylor (1991).

For testing of the implemented material law we introduce the beam considered below as the first example. For the meshing we use the 4 node shell element SHELL 43 available for creep computations. This element is based on the Reissner-Mindlin type shell theory and contains $2 \times 2$ Gauss points in the plane and five integration points through the thickness. The automatical time stepping feature with a minimum time step $0.1 \mathrm{~h}$ has been used. Figure 5 shows the solutions with respect to different meshes. The convergent solution (case 6) has been obtained with 200 elements and after 96 time steps. This solution has a very good agreement with the solution obtained using the Ritz method, Fig. 2. Similarly to the sensitivity of the solution to the degree of the polynomial discussed below the mesh sensitivity of the finite element solution can be observed. The mesh justified for the elastic solution (case 1) is not fine enough. The solutions in the cases 3-6 are approximately the same in the steady state and slightly differ in the last stage. Since for the large structures including stress singularities is difficult to test the mesh sensitivity performing the whole creep damage calculations one can conclude that convergent solution for the steady state creep which does not require many time steps, can provide an adequate (at least qualitatively correct) solution within the framework of continuum damage mechanics. 

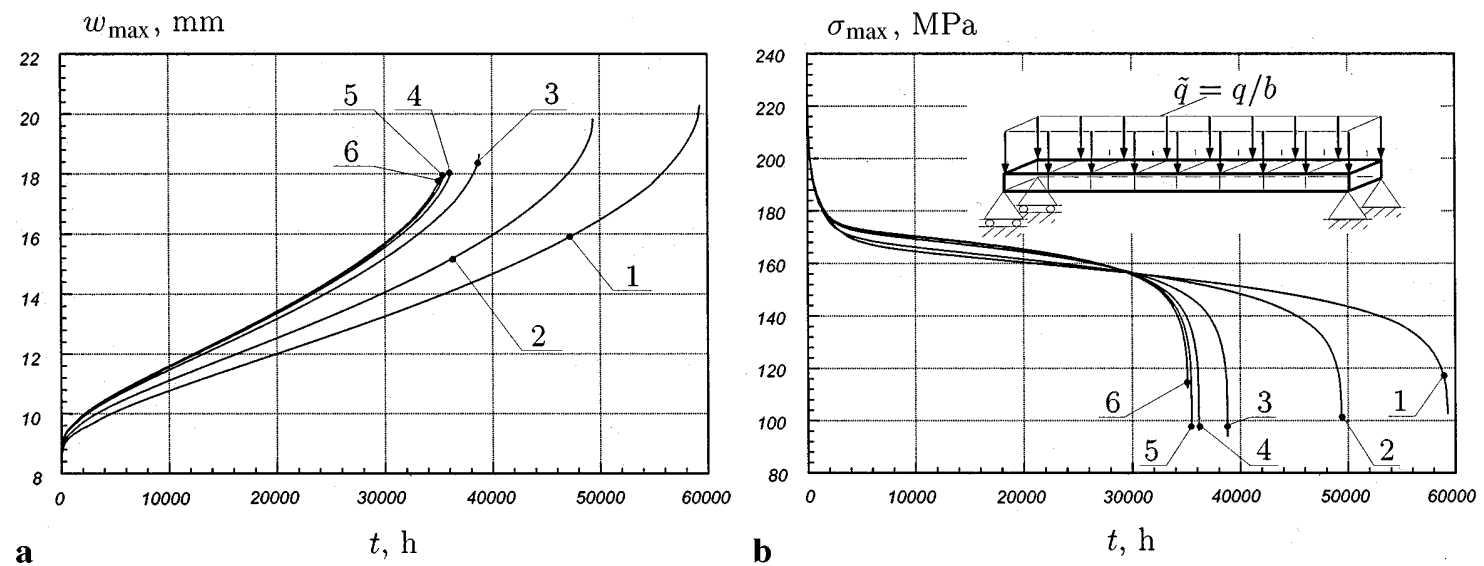

Fig. 5a, b. Solutions for a Bernoulli beam using the ANSYS code with plastic shell elements SHELL 43: a time variation of maximum deflection; b time variation of maximum stress, $1-8$ elements, $2-10$ elements, $3-20$ elements, $4-40$ elements, 5 - 80 elements, $6-200$ elements

In the previous examples we assumed the damage evolution to be the same by tensile or compressive loading. The next example illustrate the time dependent solutions of a beam setting damage rate to zero in the Gauss points

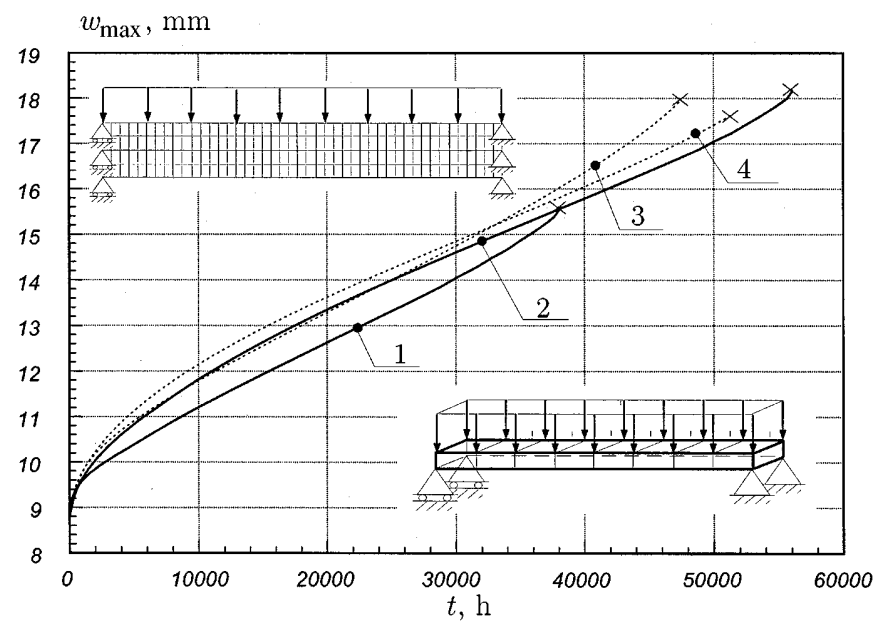

Fig. 6. Time dependent deflection of a beam: 1 - SHELL 43, 2 PLANE 42, 3 - Ritz method with 5 Gauss points for thickness integration, 4 - Ritz method with 9 Gauss points for thickness integration with negative maximum principal stress. In order to compare the influence of the element type on the creep damage solutions we performed the calculations using the element SHELL 43 with 80 elements and the 4-node plane stress element PLANE 42 meshing the beam with 80 elements along the beam axis and 4 elements along the normal axis. Figure 6 shows the time dependent solutions for maximum deflection obtained with shell elements, plane stress elements and using the Ritz method. It can be seen that if the sensitivity of material damage to the kind of stress state is taken into account results obtained by shell and plane elements substantively differ. The difference in the life-time prediction is approximately $30 \%$. For the comparison two convergent solutions based on the Ritz method applied for a beam with the Bernoulli assumptions are plotted. The first solution is obtained with 5 Gauss points for the thickness integration (curves 3), and the second with 9 Gauss points (curves 4). Both the solutions are obtained with the same number of the shape functions, $N=8, M=8$ in Eqs. (25). The solution with 9 Gauss points is in better agreement with the plane stress solution which is seen particularly on the time variation of maximum negative stress, Fig. $7 \mathrm{~b}$. The solution with 5 Gauss points agree better with the solution with the shell element. Since the element SHELL 43 contains 5 Gauss
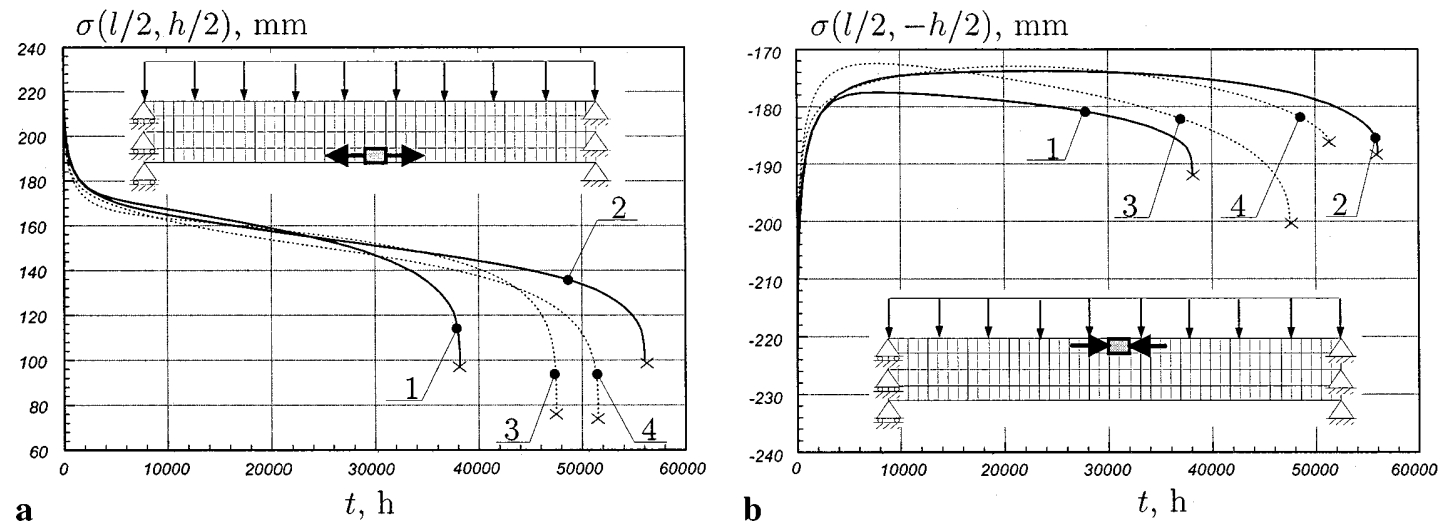

Fig. 7a, b. Time dependent stresses for a beam: a positive stress on the bottom side; $\mathbf{b}$ negative stress on the top side, curve symbols see Fig. 6 
points for the thickness integration (see ANSYS User's Manual Volume I-IV, 1994), one can conclude that more accurate thickness integration should be performed using shell elements in creep damage computations with material models reflecting different sensitivity of damage evolution to tensile and compressive loading.

\section{4}

\section{Creep-damage of plates in bending}

As the second mechanical model for the verification of user material subroutine let us introduce the thin plate problem. Analogously to the Bernoulli beams we formulate the variational problem for the thin plate using the Kirchhoff assumptions. Further we include geometrical nonlinear terms for consideration finite deflection in the sense of the von Kármán plate theory. In Altenbach and Naumenko (1997) and Altenbach et al. (1997a) we discussed the governing equations of the plate theory including creep strains and damage. The variational functional in the mixed form can be written as follows $(i, j, k, l=1,2)$

$$
\begin{gathered}
\Pi(\Phi, w)=\frac{1}{2} \int_{A}\left[-\left(g_{i j k l}^{I}\right)^{-1} N_{i j}(\Phi) N_{k l}(\Phi)\right. \\
\left.+g_{i j k l}^{I I} \mu_{i j}(w) \mu_{k l}(w)\right] \mathrm{d} A \\
+\int_{A} \frac{1}{2} N_{i j}(\Phi) \mu_{i j}(w) w \mathrm{~d} A \\
-\int_{A}\left[q w+\left(g_{i j k l}^{I}\right)^{-1} N_{i j}^{\mathrm{cr}} N_{k l}(\Phi)\right. \\
\left.+M_{i j}^{\mathrm{cr}} \mu_{i j}(w)\right] \mathrm{d} A,
\end{gathered}
$$

where $w$ is the deflection function, $\mu_{i j}$ denote the curvature changes and twist of the midsurface, $N_{i j}$ are membrane forces, $A$ is the plate area and $h$ is the plate thickness. $N_{i j}$ can be expressed with the Airy stress function $\Phi$ and $\mu_{i j}$ with the deflection function as follows

$N_{11}(\Phi)=\Phi_{, 22}, \quad N_{22}(\Phi)=\Phi_{, 11}, \quad N_{12}(\Phi)=-\Phi_{, 12}$, $\mu_{i j}(w)=-w_{, i j}$.

The deflection function and the Airy stress function depend on the cartesian coordinates $x, y \cdot(\ldots), 1,(\ldots), 2$ denote the derivatives with respect to $x$ and $y$. For the fixed time variable we can introduce the thickness averages of creep strains, assuming for the simplicity $\zeta=0$ in Eq. (8)

$N_{i j}^{\mathrm{cr}}=\int_{h} C_{i j k l} \varepsilon_{k l}^{\mathrm{cr}} \mathrm{d} z, \quad M_{i j}^{\mathrm{cr}}=\int_{h} C_{i j k l} \varepsilon_{k l}^{\mathrm{cr}} z \mathrm{~d} z$,

with

$$
\begin{aligned}
& g_{i j k l}^{I}=C_{i j k l} h, \quad g_{i j k l}^{I I}=C_{i j k l} h^{3} / 12, \quad i, j, k, l=1,2 \\
& \left(g_{i j k l}^{I}\right)^{-1}=\frac{1}{2 E h}\left[\left(\delta_{i k} \delta_{j l}+\delta_{i l} \delta_{j k}\right)(1+v)-2 v \delta_{i j} \delta_{k l}\right] .
\end{aligned}
$$

This simplification can be made due to the small influence of $\zeta$ on the failure times (as was shown by beam calculations) and the significant increasing of the computational efforts. Limiting our discussion to rectangular plates $\left(l_{1} \times l_{2}\right)$ we can solve the variational problem with following approximations $\left(\xi=x / l_{1}, \eta=y / l_{2}\right)$

$$
\begin{aligned}
& w(\xi, \eta)=a_{0} X_{\xi 0}^{w}(\xi) X_{\eta 0}^{w}(\eta)+\sum_{i=1}^{N} \sum_{j=1}^{N} a_{i j}^{w} X_{\xi i}^{w}(\xi) X_{\eta j}^{w}(\eta) \\
& \Phi(\xi, \eta)=\sum_{i=1}^{M} \sum_{j=1}^{M} a_{i j}^{\Phi} X_{\xi i}^{\Phi}(\xi) X_{\eta j}^{\Phi}(\eta)
\end{aligned}
$$

with the shape functions defined for the whole plate domain. The product function $X_{\xi 0}^{w}(\xi) X_{\eta 0}^{w}(\eta)$ is formulated as the elastic deflection function by given applied load. The procedure for the construction of such a function basing on the Vlasov-Kantorovich method and variational iterations is discussed in Altenbach et al. (1998a), Altenbach et al. (1998b). The functions $X_{\xi i}^{w}(\xi)$ and $X_{\xi i}^{\Phi}(\xi)$ are chosen as eigenfunctions of transversely vibrating beam

$$
\begin{aligned}
X_{\xi i}^{w}(\xi)= & \sin \mu \xi-\sinh \mu \xi \\
& -\frac{\sin \mu-\sinh \mu}{\cos \mu-\cosh \mu}(\cos \mu \xi-\cosh \mu \xi), \\
X_{\xi i}^{\Phi}(\xi)= & \sin i \pi \xi, \quad i=1,3,5, \ldots
\end{aligned}
$$

with

$\mu=\frac{2 i+1}{2} \pi, \quad i=1,3,5, \ldots$.

These functions satisfy exactly the essential boundary conditions for the edges $x=$ const

$w=0, \quad w_{11}=0, \quad n_{11}=\Phi_{.22}=0$

and $y=$ const

$w=0, \quad w_{, 2}=0, \quad n_{22}=\Phi_{, 11}=0$

The solution method for the creep problem of a plate which is similar to the above discussed procedure for a beam is discussed in detail in Altenbach and Naumenko (1997) and Altenbach et al. (1997a).

On Fig. 8 the time-dependent maximum deflection for a clamped square plate transversely loaded by $q=10 \mathrm{MPa}$ uniformly distributed on a square area is presented. The

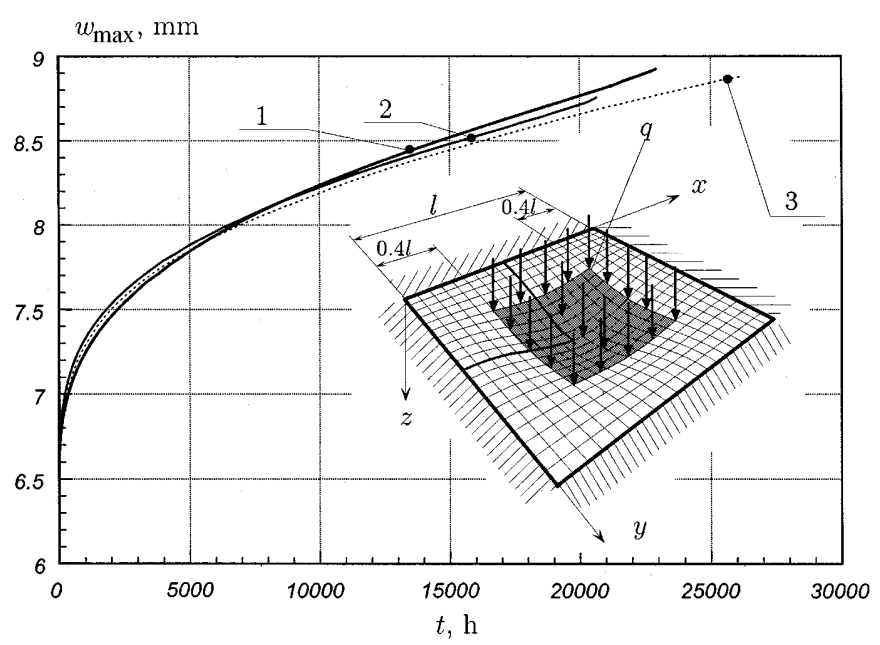

Fig. 8. Time-dependent maximum deflection of a clamped square plate: 1 - SHELL 43, 2 - SOLID 95, 3 - solution basing on the Ritz method (Kirchhoff's theory) 

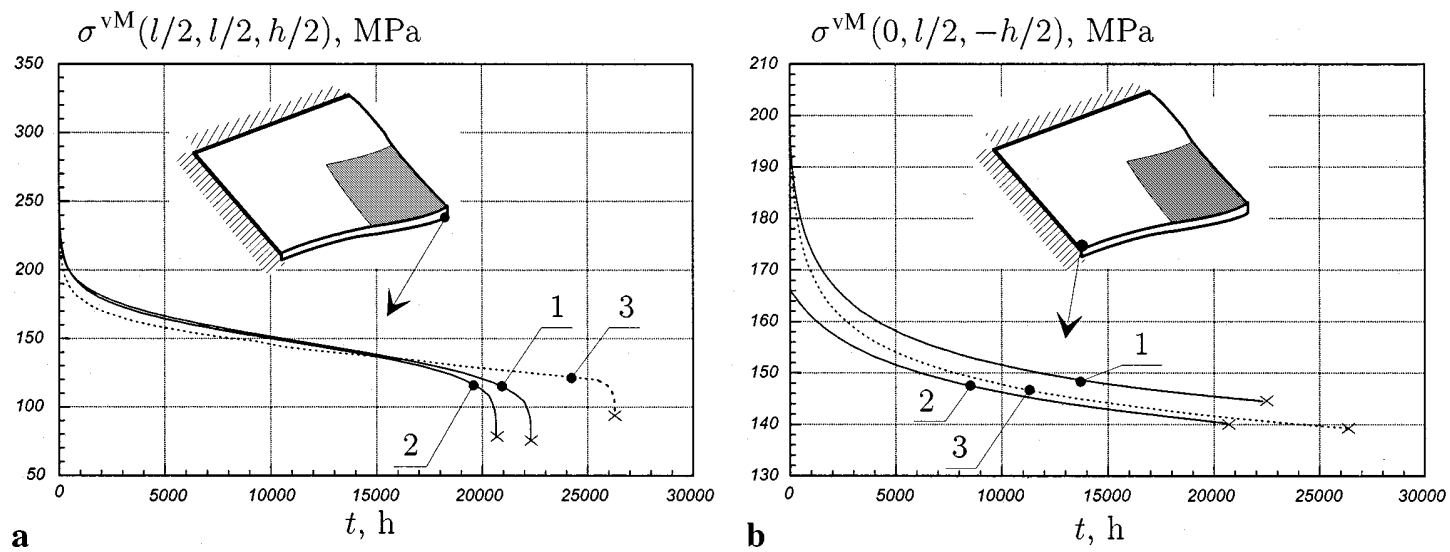

Fig. 9a, b. Time variations of the von Mises stress in two points: a midpoint of the bottom side; $\mathbf{b}$ midpoint of the clamped edge (top side), curve symbols see Fig. 8

plate length is $l=800 \mathrm{~mm}$ and the thickness $-h=27 \mathrm{~mm}$. The material constants are the same as in examples for beams. In the first example we set $\alpha=0$ in Eqs. (4) assuming the damage evolution to be dependent on the von Mises stress only. This dependency assume the same damage rate for the tensile and compressive loading. The first solution (Fig. 8, curve 1) has been obtained using the rectangular shell elements and a $20 \times 20$-element mesh for a quarter of the plate. The second solution (Fig. 8, curve 2) is based on 20-nodes solid element (3D) and $20 \times 20$ elements for a quarter of the plate and 3 elements in the thickness direction. The third solution is obtained using the Ritz solution technique. All solutions are in a good agreement. In addition, the good agreement of these three solutions can be seen on Fig. 9 for time variation of the von Mises stress plotted in two Gauss points (in the middle of the plate, bottom side and in the middle of the clamped edge, top side). In the second example we set $\alpha=1$ in the material model (4) assuming the $\sigma_{I}$-dependency of the damage evolution.

Figure 10 shows three convergent solutions for the time-dependent maximum deflection obtained using shell elements, solid elements and the Ritz method. The differences between the cases 1 (SHELL 43, Reissner-Mindlin type element) and 3 (Ritz method, Kirchhoff theory) can be explained as the influence of the shear correction. This influence is possible due to the differences of damage evolution by tensile and compressive loading which induce a nonsymmetric deformation in the thickness direction. Since the elastic solutions are the same for the starting time, the time dependent deflection obtained using the Reissner-Mindlin theory is greater during the creep process. The failure times predicted using finite element concept with shell elements agree with that basing on the Kirchhoff plate theory and the Ritz method. In contrast, the result obtained with solid elements significantly differs from both the previous cases (curve 2). The explanation of this is presented in Fig. 11, where the maximum principal stress is plotted as a function of time in two Gauss points of the plate domain. Since both the plate theories yield approximately the same values of the maximum principal stress during the whole creep process, the $3 \mathrm{D}$ theory yields the principal stress in the midpoint of the

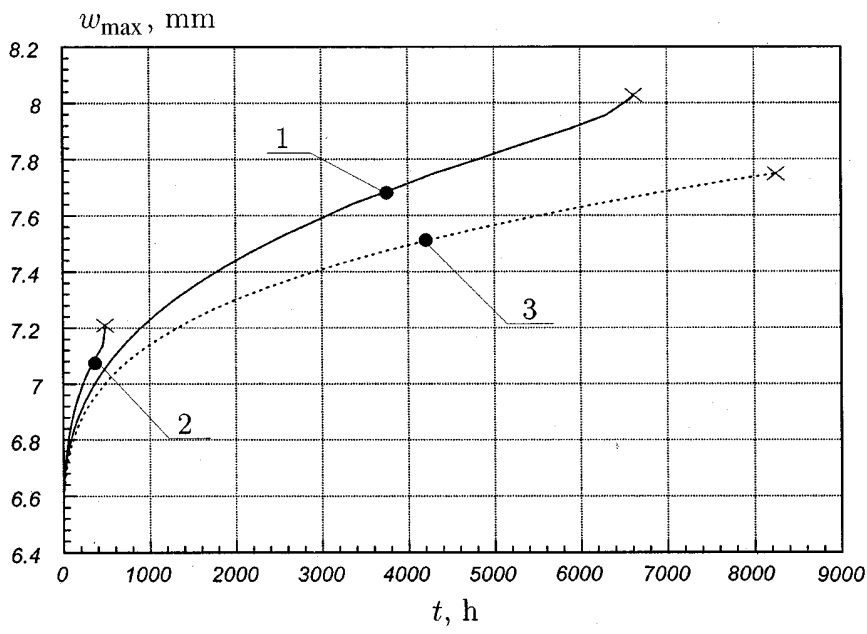

Fig. 10. Time-dependent deflection of a clamped square plate, with $\sigma_{I}$ damage criterion: 1 - SHELL 43, 2 - SOLID 95, 3 solution based on the Ritz method (Kirchhoff theory)

plate edge which is too high comparing with those for plate theories.

For the further explanation we refer to Okumura and Oguma (1998) where a closed series solution of 3D equations of elasticity for a clamped rectangular plate is discussed. The authors have shown that at the upper (lower) corners of the plate edge the 3D elasticity equations yield a singular solution for the normal stresses (the shear stresses are finite). The finite element result confirms the closed solution yielding to high values of normal stresses and the corresponding value of the maximum principal stress lead to a short failure time. Further as shown by Okumura and Oguma (1998) $\sigma_{y}=\sigma_{z}$ in the middle point of the plate. In contrast, the plate theories yield either zero or small values of the normal stress $\sigma_{z}$ compared with $\sigma_{x}$ and $\sigma_{y}$. Since $\sigma_{x}, \sigma_{y}$ and $\sigma_{z}$ are much greater than the shear stresses, the principal stresses are approximately equal to the normal stresses $\sigma_{\mathrm{I}} \approx \sigma_{x}, \sigma_{\mathrm{II}} \approx \sigma_{y}$ and $\sigma_{\mathrm{III}} \approx \sigma_{z}$. Because $\sigma_{\mathrm{II}}=\sigma_{\mathrm{III}}$ in the midpoint of the clamped edge Okumura and Oguma (1998) we can estimate the von Misses stress a $\sigma^{\mathrm{vM}}=\sigma_{\mathrm{I}}-\sigma_{\mathrm{III}}$. Since the finite element solution yield large but nonsingular values of $\sigma_{\mathrm{I}}$ and $\sigma_{\mathrm{III}}\left(\sigma_{\mathrm{I}}>\sigma_{\mathrm{III}}\right)$ the von Mises stress $\sigma^{\mathrm{vM}} \ll \sigma_{\mathrm{I}}$. This gives the explanation that 

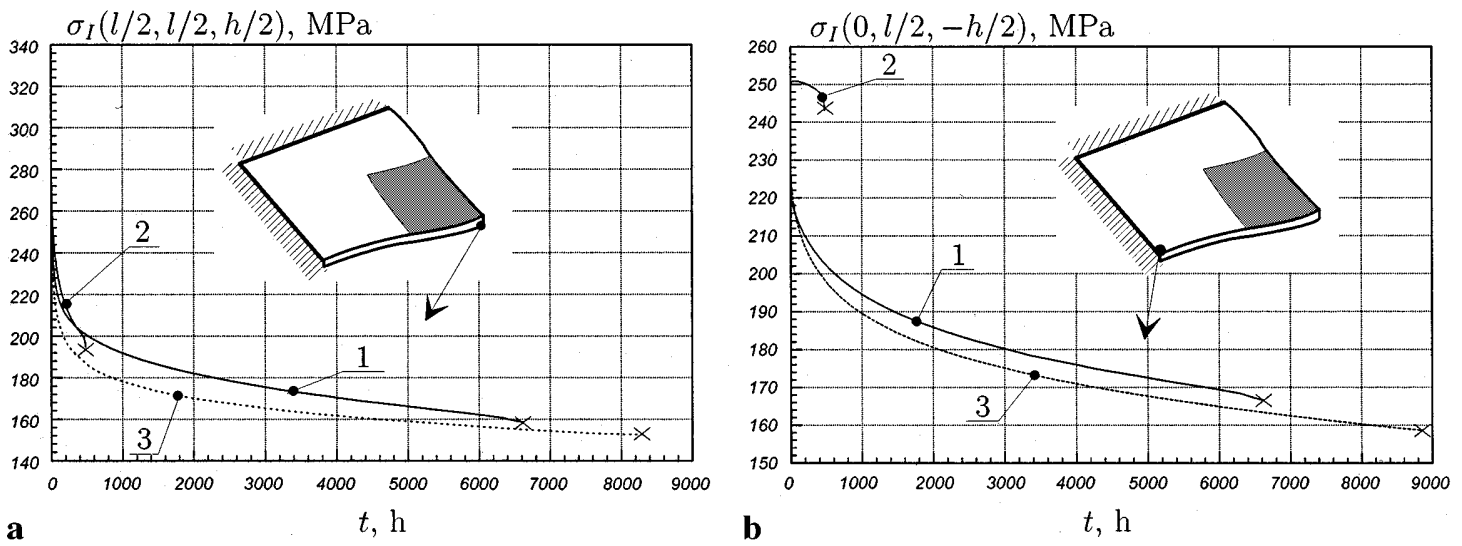

Fig. 11a, b. Time variation of the first principal stress in two points of a plate: a midpoint of the bottom side; $\mathbf{b}$ midpoint of the clamped edge (top side), curve symbols, see Fig. 10

the von Mises stress in the first example using 3D approach is less then that obtained using plate theories (Fig. 9b). In contrast, the first principal stress in the midpoint at the plate edge using $3 \mathrm{D}$ mesh is much greater. Therefore the use of $\sigma_{I}$ in damage evolution equation yields to a very short failure time comparing with those for plate models.

\section{5}

\section{Conclusions}

The standard FE-code ANSYS which allows elastic and inelastic analysis of beams, plates and shells taking into account different constitutive equations was used for the long-term predictions in the case of creep-damage behaviour of the material at stationary elevated temperatures. The damage evolution is included using KachanovRabotnov's scalar damage parameter and Norton's creep law for the creep process. The creep-damage material model is incorporated into the ANSYS code by writing a user creep material subroutine.

The accuracy of the creep-damage finite element predictions in thinwalled structures was estimated comparing with solutions based on the Ritz method. As examples the beam and the plate in bending problems were discussed in detail. Various numerical tests verify the implemented user material subroutine and show the sensitivity of the long-term predictions for beams and plates to the mesh sizes and the element types. Basing on some analytical estimations, solutions with the Ritz method and finite element solutions we conclude the following:

1. The approximations or meshes justified for the elastic solutions using displacement based variational methods cannot be used for the creep damage analysis.

2. The mesh established basing on a convergent steady state creep solutions can be used for the continuum damage mechanics analysis of thinwalled structures in bending.

These conclusions can be related to the finite element concept in general applying any finite element code.

If the material behaviour depends on the kind of stress state (e.g., different damage rate to tensile and compressive stresses) the results for beams and plates are sensitive to element type. First the structure mechanics equations for beams, plates and shells have to be based on refined cross section kinematics (e.g., shear deformable theories). This has been demonstrated on plates using the Kirchhoff's and Reissner-Mindlin's theories. Second, let us note, that the finite element studies in the case of plates have been based on the Reissner-Mindlin type elements available in the ANSYS code for plasticity and creep simulations. Further investigations should be directed to the performance of shell or plate theories and corresponding types of finite elements available in other commercial systems (e.g. ABAQUS) in the long-term predictions using continuum damage mechanics material models.

\section{References}

Altenbach H (1999) Classical and non-classical creep models. In: Altenbach H, Skrzypek J (eds.), Creep and Damage in Materials and Structures. Springer-Verlag, Wien et al., pp. 45-94

Altenbach H, Altenbach J, Naumenko K (1998a) Ebene Flächentragwerke. Springer-Verlag, Berlin u.a.

Altenbach H, Morachkovsky O, Naumenko K, Sychov A (1997a) Geometrically nonlinear bending of thin-walled shells and plates under creep-damage conditions. Arch. Appl. Mech. 67:339-352

Altenbach H, Naumenko K (1997) Creep bending of thin-walled shells and plates by consideration of finite deflections. Comp. Mech. 19:490-495

Altenbach J, Altenbach H, Naumenko K (1997b) Lebensdauerabschätzung dünnwandiger Flächentragwerke auf der Grundlage phänomenologischer Materialmodelle für Kriechen und Schädigung. Technische Mechanik 17:353-364

Altenbach J, Naumenko K, Naumenko V (1998b) Analysis of rectangular thin plates and plate structures basing on the Vlasov's variational procedure. CAMES 5:115-128

ANSYS User's Manual Volume I-IV (1994) Swanson Analysis Systems, Inc.

Becker AA, Hyde TH, Xia L (1994) Numerical analysis of creep in components. J. Strain Anal. 29:27-34

Bialkiewicz J, Mika P (1995) Computer analysis of damage development in rectangular plates. Eng. Trans. 43:71-82

Bodnar A, Chrzanowski M (1994) Cracking of creeping plates in terms of continuum damage mechanics. Mech. Teor. i Stos. 32:31-41

Boyle JT, Spence J (1983) Stress analysis for creep. Butterworths, London 
Chosh RN, McLean M (1992) High temperature deformation in engineering alloys-modelling for strain of load control. Acta Metall. Mater. 40:3075-3083

Fleig T (1996) Lebensdaueranalyse unter Berücksichtigung viskoplastischer Verformung und Schädigung mit der Methode der Finite Elemente. Dissertation, Universität Karlsruhe

Hayhurst DR (1994) The use of continuum damage mechanics in creep analysis for design. J. Strain Anal. 25:233-241

Hyde TH, Xia L, Becker AA (1996) Prediction of creep failure in aeroengine materials under multi-axial stress states. Int. J. Mech. Sci. 38:385-403

Kachanov L (1986) Introduction to Continuum Damage Mechanics. Martinus Nijhoff Publishers, Dordrecht

Kowalewski ZL, Hayhurst DR, Dyson BF (1994) Mechanismbased creep constitutive equations for an aluminium alloy. J. Strain Anal. 29:309-316

Leckie F, Hayhurst D (1977) Constitutive equations for creep rupture. Acta Metall. 25:1059-1070

Lemaitre J, Chaboche JL (1990) Mechanics of Solid Materials. Cambridge University Press, Cambridge.

Liu Y, Murakami S (1998) Damage localization of conventional creep damage models and proposition of new model for creep damage analysis. JSME 41:57-65

Liu Y, Murakami S, Kanagawa (1994) Mesh-dependence and stress singularity in finite element analysis of creep crack growth by continuum damage mechanics approach. Eur. J. Mech. A Solids 13:395-417

Murakami S, Liu Y (1995) Mesh-dependence in local approach to creep fracture. Int. J. Damage Mech. 4:230-250
Nabarro FRN, de Villiers HL (1995) The Physics of Creep. Creep and Creep-resistant Alloys. Taylor \& Francis, London

Naumenko K (1996) Modellierung und Berechnung der Langzeitfestigkeit dünnwandiger Flächentragwerke unter Berücksichtigung von Werkstoffkriechen und Schädigung. Dissertation, Otto-von-Guericke Universität Magdeburg

Okumura IA, Oguma Y (1998) Series solutions for a transversely loaded and completely clamped thick rectangular plate based on the three-dimendional theory of elasticity. Arch. Appl. Mech. 68:103-121

Othman AM, Dyson BF, Hayhurst DR, Lin J (1994) Continuum damage mechanics modelling of circumferentially notched tension bars undergoing tertiary creep with physically-based constitutive equations. Acta. Metall. Mater. 42:597-611

Perrin IJ, Hayhurst DR (1994) Creep constitutive equations for a $0.5 \mathrm{Cr}-0.5 \mathrm{Mo}-0.25 \mathrm{~V}$ ferritic steel in the temperature range 600-675 ${ }^{\circ}$ C. J. Strain Anal. 31:299-314

Pilkey W, Wunderlich W (1994) Mechanics of Structures - Variational and Computational Methods. CRC Press, Boca Raton

Rabotnov YN (1969) Creep Problems in Structural Members. North Holland, Amsterdam

Roche RL, Townley CHA, Regis V, Hübel H (1992) Structural Analysis and Available Knowledge. In: Larson LH (ed.), High Temperature Structural Design. Mechanical Engineering Publications, London, pp. 161-180

Saanouni K, Chaboche JL, Lense PM (1989) On the creep crackgrowth prediction by a non-local damage formulation. Eur. J. Mech. A Solids 8:437-459

Zienkiewicz OC, Taylor RL (1991) The Finite Element Method. McGraw-Hill, London 